\title{
Registro Brasileiro de Marcapassos: escolha do modo de estimulação no ano de 1999
}

\author{
Roberto COSTA*, Luiz Antonio Castilho TENO**, Antonio Amauri GROPPO***, Vicente ÁVILA NETO**, \\ Roberto BELTRAME ${ }^{\star \star \star *}$, Carla Pintas MARQUES ${ }^{\star \star \star *}$, Paulo Roberto Slud BROFMAN"
}

RBCCV 44205-512

Costa R, Teno L A C, Groppo A A, Ávila Neto V, Beltrame R, Marques C P, Brofman P R S - Registro Brasileiro de Marcapassos: escolha do modo de estimulação no ano de 1999. Rev Bras Cir Cardiovasc2000; 15(3): 263-71.

RESUMO: O Registro Brasileiro de Marcapassos (RBM) é uma base de dados nacional que visa a coletare divulgar informações concernentes aos procedimentos relacionados com a estimulação cardíaca artificial no Brasil. Este trabalho apresenta os resultados do sexto ano de operação do RBM, que vai de 1ㅇ de janeiro a 31 de dezembro de 1999 e teve como objetivo principal comparar os dados clínicos dos pacientes submetidos a implante de marcapasso ventricular aos dos pacientes que receberam marcapasso atrioventricular. Nesse período, foram reportados 11.048 procedimentos, perfazendo 8141 (73,7\%) implantes iniciais e 2907 (26,3\%) reoperações. Foram implantados $6779(61,4 \%)$ marcapassos unicamerais e $4258(38,6 \%)$ bicamerais. Dos implantes de câmara única, apenas $99(0,9 \%)$ foram atriais. A relação média nacional entre implantes ventriculares e atrioventriculares foi de 1,6: 1. Pela análise dos dados apresentados para os primeiros implantes de marcapasso, foi possível verificar fatores que influenciaram a escolha do modo de estimulação, como: 1) a região onde o hospital está instalado;2) a idade do paciente; 3 ) a classe funcional e 4) o distúrbio da condução do paciente.

Diferenças Regionais: A região centro-oeste diferiu das demais, por apresentar maior número de implante atrioventriculares que ventriculares (relação $A V / V V I=1,5$ ). Nas demais regiões, foram implantados mais marcapassos ventriculares que dupla-câmara com relações AV / VVI variando de 0,78 na região sudeste a 0,51 na região sul.

Idade: Na faixa etária de 21 a 60 anos, houve mais implantes bicamerais (relação $A V / V V I=1,7$ dos 21 aos 40 anos e 1,25 dos 41 aos 60). Nas demais faixas etárias, o número de implantes ventriculares foi maior, com relação VVI/AV variando de 1,3: 1 nas duas primeiras décadas da vida a 3,2: 1 na faixa etária maior que 81 anos.

Classe Funcional: apenas nos pacientes de classe funcional I o número de marcapassos bicamerais excedeu o número de ventriculares (relação $A V / V V I=1,4$ ). Nos pacientes com sintomas de insuficiência cardíaca prévia, o número de implantes ventriculares foi maior com relação VVI / AV de 1,2: 1 na classe II; de 1,5: 1 na classe III e de 1,9: 1 na classe IV.

Distúrbio do Sistema Éxcito-Condutor: Pacientes portadores de doença do nó sinusal receberam aproximadamente duas vezes mais marcapassos fisiológicos que ventriculares (relação AV/VVI=2,1:1). Nos portadores de bloqueios do ${ }^{2}$ o $\mathrm{grau}$, a relação VVI/AV foi de $1,2: 1$, nos portadores de bloqueios totais, foi $1,8: 1$ e nos pacientes portadores de flutter ou fibrilação atrial 10: 1.

Outros Fatores Analisados: Sintomas pré-operatórios, etiologia do distúrbio da condução e sexo do paciente não estiveram relacionados a diferenças no modo de estimulação escolhido.

DESCRITORES: Estimulação cardíaca artificial, coleta de dados. Sistemas de gerenciamento de base de dados, Brasil. Marcapassos cardíacos, implante, coleta de dados. Registro Brasileiro de Marcapassos; Ministério da Saúde / Sociedade Brasileira de Cirurgia Cardiovascular. Departamento de Estimulação Cardíaca / Sociedade Brasileira de Cirurgia Cardiovascular. Marcapasso artificial. Estimulação cardíaca artificial, métodos. Registros médicos, Brasil. Bases de dados, Brasil. Marcapasso artificial, coleta de dados, Brasil.

Trabalho realizado pelo Ministério da Saúde / Sociedade Brasileira de Cirurgia Cardiovascular, São Paulo, SP. Brasil.

Recebido para publicação em agosto de 2000.

* Coordenador do Registro Brasileiro de Marcapassos - RBM.

** Presidente do Departamento de Estimulação Cardíaca Artificial da SBCCV (DECA).

*** Membro da Diretoria do Departamento de Estimulação Cardíaca Artificial da SBCCV (DECA).

**** Representante do Ministério da Saúde.

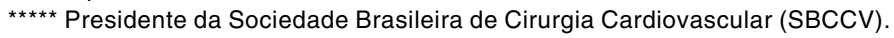

Endereço para correspondência: Roberto Costa. Rua Beira Rio, 45, cj. 73. São Paulo, SP, Brasil. CEP 04548-050. Tel. (11) 3842-1352. e-mail: marcapasso@osite.com.br 
Costa R, Teno L A C, Groppo A A, Ávila Neto V, Beltrame R, Marques C P, Brofman P R S - Registro Brasileiro de Marcapassos: escolha do modo de estimulação no ano de 1999. Rev Bras Cir Cardiovasc 2000; 15(3): 263-71.

\section{INTRODUÇÃO}

Os critérios comumente observados para escoIha do modo de estimulação cardíaca artificial baseiam-se em três pontos fundamentais:

a) no conhecimento da fisiologia cardíaca, a partir do qual, objetiva-se, sempre que possível, a manutenção ou a reconstituição do sincronismo atrioventricular;

b) na relação custo / benefício, que implicaria na compensação do gasto, inicialmente mais elevado dos sistemas atrioventriculares, pela diminuição do número de internações e do uso de medicamentos no longo prazo;

c) nas evidências obtidas na literatura de que o implante de um sistema mais "fisiológico" proporciona menor mortalidade, melhor qualidade de vida e menor índice de complicações que o uso de um marcapasso ventricular.

Tendo como base o melhor desempenho hemodinâmico dos pacientes portadores de marcapassos que preservam a seqüência atrioventricular, os diversos consensos sobre estimulação cardíaca artificial têm recomendado a utilização de marcapassos atriais ou atrioventriculares sempre que as câmaras atriais apresentem estabilidade elétrica $(1,2)$.

Vários autores têm trabalhado no sentido de projetar a diferença do custo de sistemas uni e bicamerais no curto e no longo prazo ${ }^{(3,4)}$. SUTTON \& BOURGEOIS, em 1996, concluíram que após três anos de seguimento, o custo do tratamento de pacientes portadores de marcapassos bicamerais é menor que o de sistemas ventriculares, tanto na doença do nó sinusal quanto nos bloqueios da condução atrioventricular (4).

As evidências de que os sistemas baseados na contração atrial permitem maior sobrevida e menor número de complicações que os marcapassos ventriculares, entretanto, são escassas. A maioria dos trabalhos encontrados na literatura são calcados em análises retrospectivas, sujeitas a críticas. Os poucos trabalhos prospectivos têm analisado apenas a doença do nó sinusal (5-10).

O objetivo do presente relato é apresentar os dados obtidos pelo RBM no ano de 1997 apresentando-os a partir do modo de estimulação escolhido.

\section{MÉTODO}

\section{Coleta e Processamento das Informações}

O Registro Brasileiro de Marcapassos é adaptado à realidade brasileira, e é compatível com o
"European Pacemaker Registration Card". Um único documento, em quatro vias, substitui a "ficha de implante" dos diversos fornecedores, padronizandoa em todo o território nacional; uma via fica arquivada no prontuário do paciente, a segunda fica com a empresa fornecedora do marcapasso, uma terceira vai para o órgão pagador, que é o Sistema Único de Saúde (SUS) ou empresas seguradoras e uma via é remetida ao RBM, responsável pelo processamento dos dados.

Uma portaria do Ministério da Saúde (Vigilância Sanitária) regulamenta que todo o gerador de pulsos comercializado do Brasil deve conter esse formulário.

As informações são organizadas no formulário constando de: 1) dados pessoais do paciente; 2) dados clínicos; 3 ) dados cirúrgicos e 4) dados sobre o sistema de estimulação implantado e/ou removido além de 5) fechamento do arquivo. Os dados repetitivos como Cadastro de Médicos, de Hospitais, de Empresas Seguradoras e de Produtos (geradores e eletrodos) compõem os arquivos de suporte para o cadastramento, e contêm informações completas sobre os diferentes setores que compõem o RBM.

O Registro Brasileiro de Marcapassos utiliza software gerenciador do banco de dados já foi apresentado anteriormente, e foi modificado recentemente e redesenhado em Visual Basic 5.0, permitindo que o trabalho seja desenvolvido integralmente em ambiente Windows, sendo dedicado à manipulação de dados médicos (textuais), organizados na forma de tabelas de múltipla escolha. Tem como característica principal a facilidade de inserção de dados, e a versatilidade para levantamento de dados, cujos índices são criados no momento da pesquisa. Este sistema permite a pesquisa de qualquer dado, a partir do grupo total ou de qualquer outro índice (subgrupo) já criado, atendendo às diversas necessidades de levantamento dos dados. Possui um módulo editor de relatórios que é utilizado para relatórios padronizados como os que são enviados para o Ministério da Saúde, para os hospitais ou para os médicos participantes do sistema.

Os formulários são recebidos pelo RBM por via postal. As planilhas são examinadas, classificadas e levadas à digitação. Aquelas que apresentam pequenos erros ou falhas de preenchimento são separadas, e é feito um contato por telefone ou por correio para que sejam corrigidas. O tempo médio de recebimento dos formulários é de 30 a 60 dias após o procedimento.

O RBM conta com uma funcionária responsável pela revisão, classificação e digitação dos formulários. Os recursos provêm da taxa cobrada pelo RBM 
Costa R, Teno L A C, Groppo A A, Ávila Neto V, Beltrame R, Marques C P, Brofman P R S - Registro Brasileiro de Marcapassos: escolha do modo de estimulação no ano de 1999. Rev Bras Cir Cardiovasc 2000; 15(3): 263-71.

por unidade de formulário fornecida aos distribuidores, e têm garantido a operacionalidade do Sistema.

$\mathrm{Na}$ presente publicação foram analisadas apenas os números dos campos assinalados, tendo sido excluídos os campos deixados em branco.

\section{Análise dos Resultados}

Após o cadastramento das informações, os dados referentes ao ano de 1999 foram separados e analisados: 1) quanto ao sexo e idade dos pacientes; 2) quanto às características clinicas e 3) quanto ao tipo de marcapasso implantado. Os dados referentes aos médicos e hospitais que realizaram o implante também foram analisados.

Os dados pessoais e clínicos foram agrupados segundo o tipo de marcapasso utilizado (ventricular ou atrioventricular) e apresentados em tabelas e gráficos. Os dados referentes aos implantes atriais puros foram retirados dessa análise, devido ao seu pequeno número (53 pacientes representando $0,7 \%$ das operações)

Os gráficos apresentam histogramas de freqüência dos números absolutos para todos os parâmetros analisados. No caso específico das variáveis clínicas, os gráficos de barras horizontais apresentam também valores percentuais.

A especialização de médicos e hospitais que realizaram procedimentos é analisada pelo agrupamento dos profissionais e instituições em quatro categorias: a) os que realizam menos que 10 operações de marcapasso por ano; b) entre 11 e 50; c) de 51 a 100; e d) acima de 100 procedimentos ao ano. Estes dados são apresentados em histogramas de freqüência.

\section{RESULTADOS}

\section{Dados Gerais}

Segundo as informações registradas nos formulários enviados por 216 hospitais e preenchidos por 392 médicos diferentes, no período de 1/1/99 a 31/ $12 / 99$, foram realizados 11.048 procedimentos cirúrgicos relacionados à estimulação cardíaca artificial. Desses, $8141(73,7 \%)$ foram implantes iniciais e 2907 (26,3\%), reoperações.

A distribuição dos pacientes por região brasileira, levando-se em consideração a localização do hospital, mostrou que na região Centro-Oeste foram realizadas $814(7,4 \%)$ operações; na região Nordeste, $1323(11,2 \%)$; na Norte, $32(0,3 \%)$; na Sudeste, $6511(58,9 \%)$ e na Sul, $2331(21,1 \%)$.
Verificou-se que 88 hospitais $(40,7 \%)$ realizaram menos que 10 operações; 64 hospitais $(29,6 \%)$ realizaram de 11 a 50 procedimentos; $31(14,4 \%)$ realizaram entre 51 e 100 operações; e apenas 33 $(15,3 \%)$ hospitais realizaram mais que 100 procedimentos no ano de 1999 (Figura 1).

Da mesma forma, observa-se que 209 (53,3\%) médicos realizaram menos que 10 operações; 112 $(28,6 \%)$ profissionais realizaram de 11 a 50 procedimentos; $46(11,7 \%)$ realizaram entre 51 e 100 operações; e apenas 25 (6.40\%) especialistas realizaram mais que 100 procedimentos em 1999 (Figura 2).

\section{Implantes Iniciais}

Analisando-se os dados referentes aos Implantes Iniciais de Marcapasso, verificou-se que sistemas de câmara única foram implantados em 4713

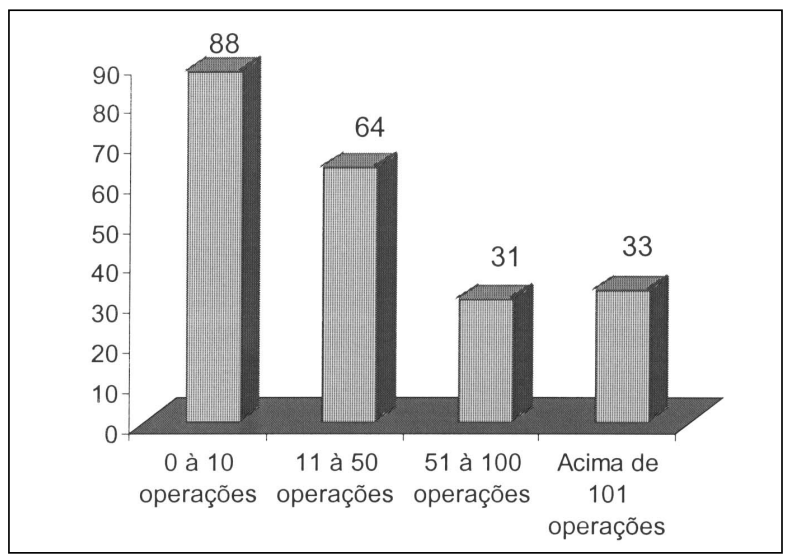

Fig. 1 - Distribuição dos hospitais que realizaram os implantes, agrupados por número de procedimentos realizados no ano de 1999.

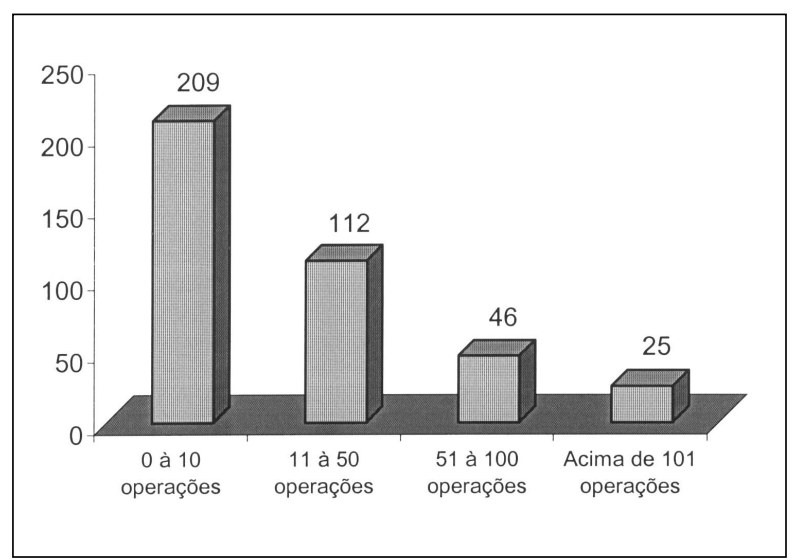

Fig. 2 - Distribuição dos médicos que realizaram os implantes, agrupados por número de procedimentos realizados no ano de 1999. 
Costa R, Teno L A C, Groppo A A, Ávila Neto V, Beltrame R, Marques C P, Brofman P R S - Registro Brasileiro de Marcapassos: escolha do modo de estimulação no ano de 1999. Rev Bras Cir Cardiovasc 2000; 15(3): 263-71.

$(57,9 \%)$ pacientes e de dupla câmara em 3428 $(42,1 \%)$. Dos marcapassos unicamerais, apenas 53 $(0,7 \%)$ eram atriais (Figura 3 ). A Figura 4 mostra a distribuição dos implantes iniciais nas diferentes regiões brasileiras.

O sexo masculino foi referido em $2344(50,4 \%)$ pacientes submetidos a implantes ventriculares, e em $1785(52,2 \%)$ dos atrioventriculares. O sexo era feminino em $2306(49,6 \%)$ pacientes submetidas a implantes ventriculares e em 1637 (47,8\%), nos atrioventriculares (Figura 5).

A análise da idade dos pacientes mostrou que, dos pacientes submetidos a implante ventricular, 61 $(1,5 \%)$ apresentavam de 1 a 20 anos de idade; 139 $(3,4 \%)$, de 21 a $40 ; 638(15,4 \%)$, de 41 a $60 ; 2331$ $(56,2 \%)$, de 61 a 80 ; e $976(23,5 \%)$, estavam acima de 81 anos. No grupo de pacientes submetidos a implantes de dupla-câmara, $46(1,5 \%)$ apresentavam de 1 a 20 anos de idade; $238(7,8 \%)$, de 21

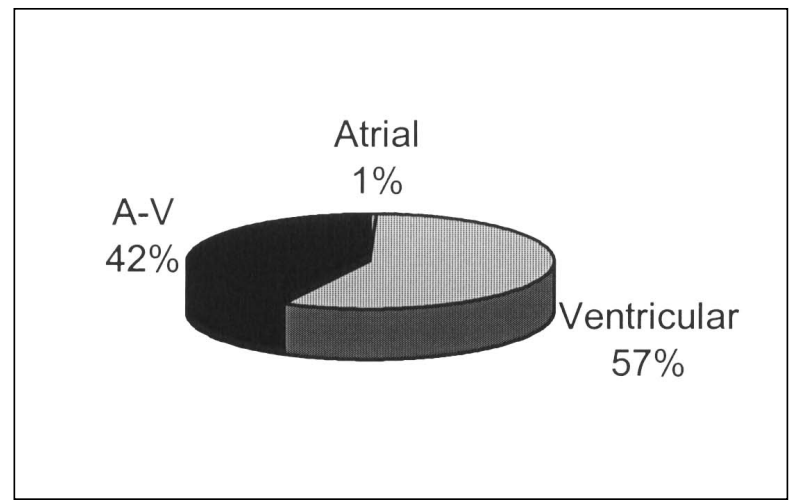

Fig. 3 - Tipo de marcapasso utilizado nos pacientes submetidos a primeiro implante.

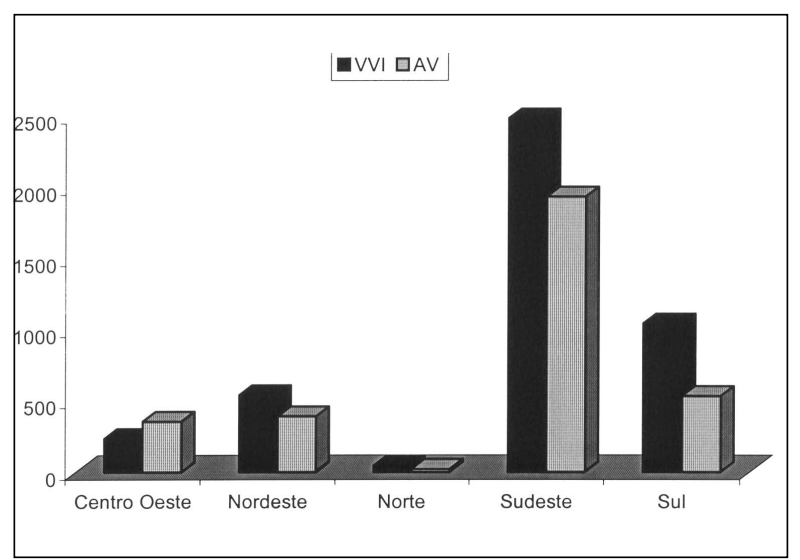

Fig. 4 - Modos de estimulação utilizados nos implantes iniciais, agrupados segundo a região brasileira onde o procedimento foi realizado. a $40 ; 799(26,0 \%)$, de 41 a $60 ; 1680(54,7 \%)$, de 61 a 80 ; e $307(10,0 \%)$, apresentavam mais que 80 anos (Figura 6).

Nos pacientes submetidos a implante ventricular, a indicação clínica para o implante de marcapasso foi justificada por síncopes, pré-síncopes ou tonturas em $3608(77,4 \%)$, pelo achado de bradicardia em $265(5,7 \%)$, por insuficiência cardíaca congestiva em $288(6,2 \%)$, por taquicardia em $84(1,8 \%)$, sendo relatados outros sintomas e sinais em $58(1,8 \%)$ enfermos. Nos implantes atrioventriculares, a referência de sintomas de baixo fluxo cerebral foi feita em $2350(68,6 \%)$ pacientes, de bradicardia em 234 $(6,8 \%)$, de insuficiência cardíaca congestiva em 261 $(7,6 \%)$, de taquicardia em $78(2,3 \%)$ e outras causas em $43(1,3 \%)$ (Figura 7 )

A classificação funcional para insuficiência cardíaca congestiva (N.Y.H.A.) mostrou que 259 (5,6\%) pacientes submetidos a implante ventricular e 368

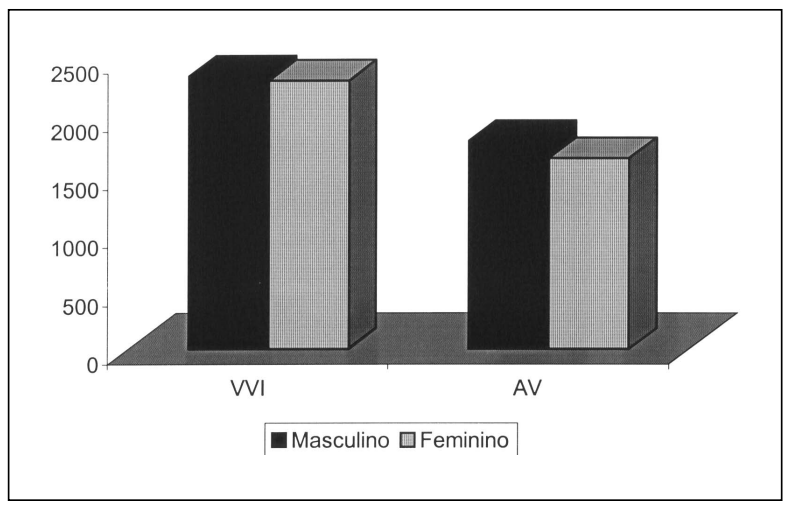

Fig. 5 - Modos de estimulação utilizados nos implantes iniciais, agrupados segundo o sexo dos pacientes.

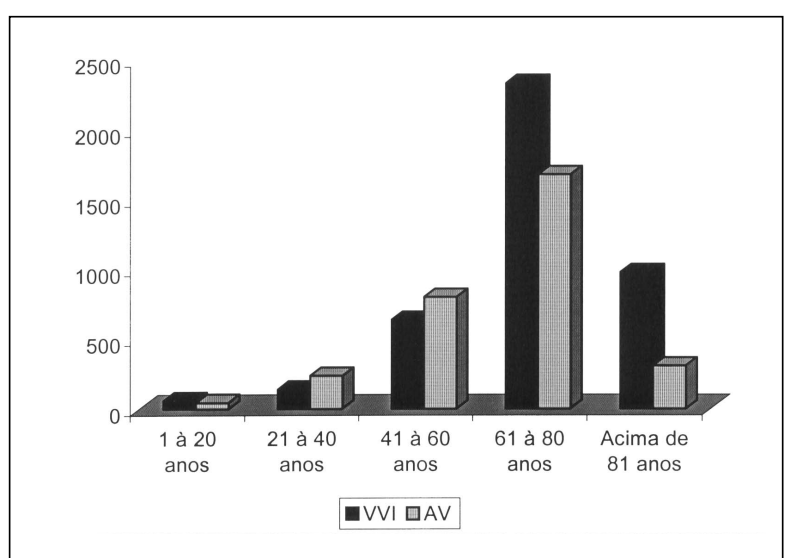

Fig. 6 - Modos de estimulação utilizados nos implantes iniciais, agrupados segundo a faixa etária dos pacientes. 
Costa R, Teno L A C, Groppo A A, Ávila Neto V, Beltrame R, Marques C P, Brofman P R S - Registro Brasileiro de Marcapassos: escolha do modo de estimulação no ano de 1999. Rev Bras Cir Cardiovasc 2000; 15(3): 263-71.

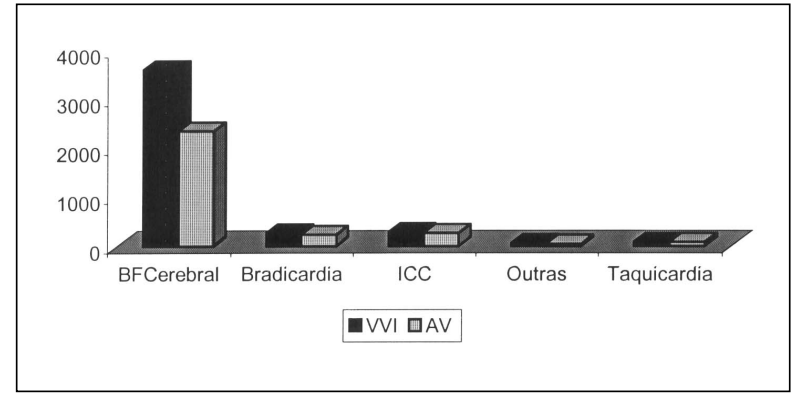

Fig. 7 - Modos de estimulação utilizados nos implantes iniciais, agrupados segundo a indicação clínica para o implante de marcapasso.

$(10,7 \%)$ dos bicamerais eram assintomáticos, e que $545(11,7 \%)$ submetidos a implante ventricular e 438 $(12,8 \%)$ dos atrioventriculares apresentavam sintomas aos grandes esforços. Na classe funcional III estavam 1999 (42,9\%) pacientes submetidos a implante ventricular e 1342 (39,1\%) submetidos a dupla-câmara. Sintomas em repouso foram reportados em 1459 (31,3\%) dos implantes ventriculares e em $762(22,2 \%)$ dos atrioventriculares. (Figuras $8 \mathrm{a}$ e $8 \mathrm{~b})$.

Os distúrbios do ritmo cardíaco que justificaram o implante de marcapasso foram, para os implantes ventriculares, o bloqueio atrioventricular do segundo

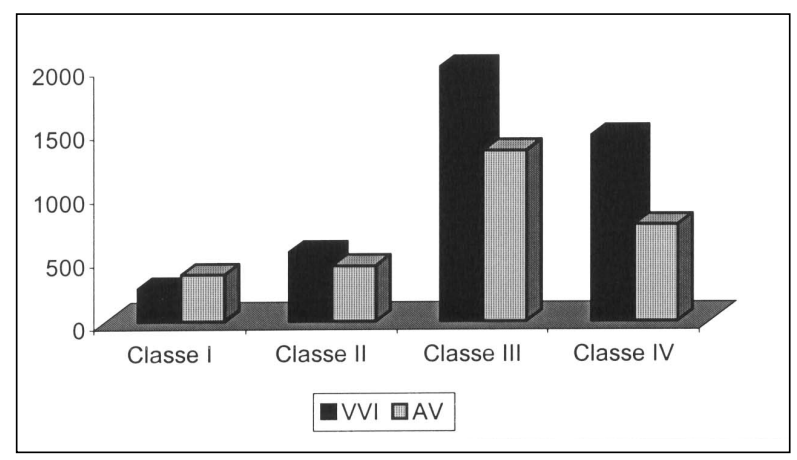

Fig. 8a - Modos de estimulação utilizados nos implantes iniciais, agrupados segundo a classificação funcional dos pacientes para insuficiência cardíaca congestiva.

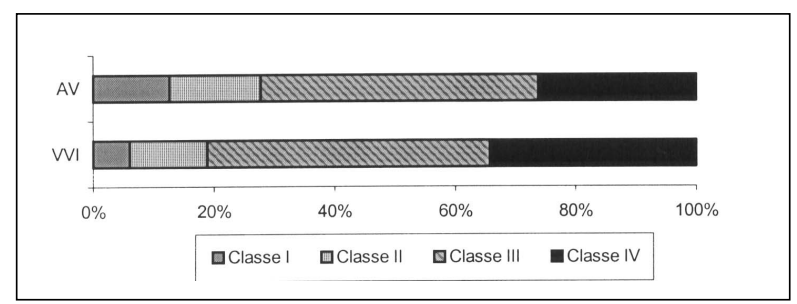

Fig. 8b - Modos de estimulação utilizados nos implantes iniciais, agrupados segundo a classificação funcional dos pacientes para insuficiência cardíaca congestiva. grau em 528 (11,3\%) pacientes; o bloqueio atrioventricular total em $2590(55,6 \%)$, os bloqueios fasciculares em 55 (1,2\%); as várias formas da doença do nó sinusal em 370 (7,9\%), o flütter ou fibrilação atrial com baixa resposta ventricular em $692(14,8 \%)$ e outros achados em $85(1,8 \%)$ pacientes. No grupo dos pacientes submetidos a implantes bicamerais os distúrbios do ritmo cardíaco relatados foram o bloqueio atrioventricular do segundo grau em 452 (11,3\%) pacientes; o bloqueio atrioventricular total em 1449 (42,3\%), os bloqueios fasciculares em 99 (2,9\%); as várias formas da doença do nó sinusal em $761(22,2 \%)$, o flütter ou fibrilação atrial com baixa resposta ventricular em $71(2,1 \%)$ e outros achados em 134 (3,9\%) pacientes (Figuras 9a e 9b).

A etiologia atribuída ao distúrbio da condução dos pacientes submetidos a implante ventricular foi a congênita em $47(1,0 \%)$ pacientes, era desconhecida em $1271(27,3 \%)$, doença de Chagas em 938 $(20,1 \%)$, fibrose do sistema de condução em 1227 $(26,3 \%)$, intervenções médicas em $92(2,0 \%)$ e outras causas em 261 (5,6\%). Dos pacientes submetidos a implantes atrioventriculares, $38(1,1 \%)$ foram referidos como congênitos, em $779(22,7 \%)$ a etiologia foi dada como desconhecida, 811 (23,7\%) eram chagásicos, em $696(20,3 \%)$ foi relatada fibrose do

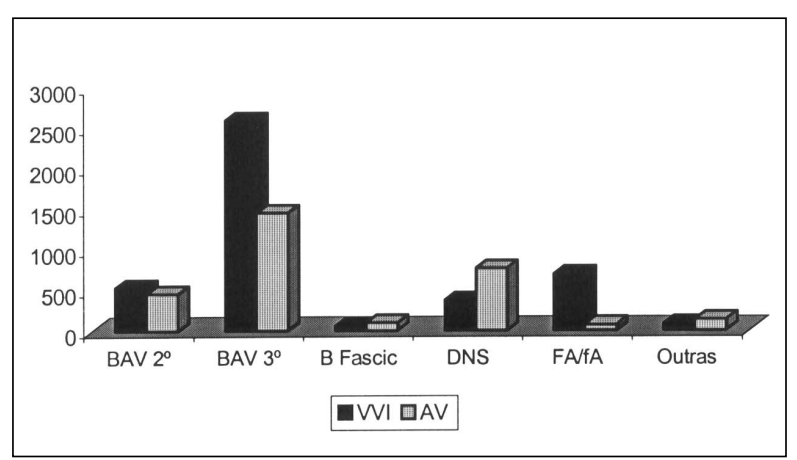

Fig. 9a - Modos de estimulação utilizados nos implantes iniciais, agrupados segundo o achado eletrocardiográfico dos pacientes.

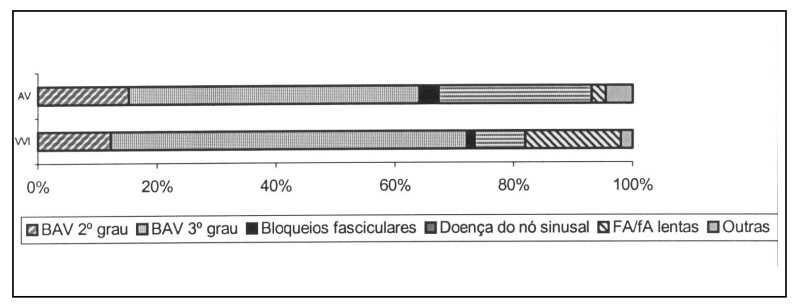

Fig. 9b - Modos de estimulação utilizados nos implantes iniciais, agrupados segundo o achado eletrocardiográfico dos pacientes. 


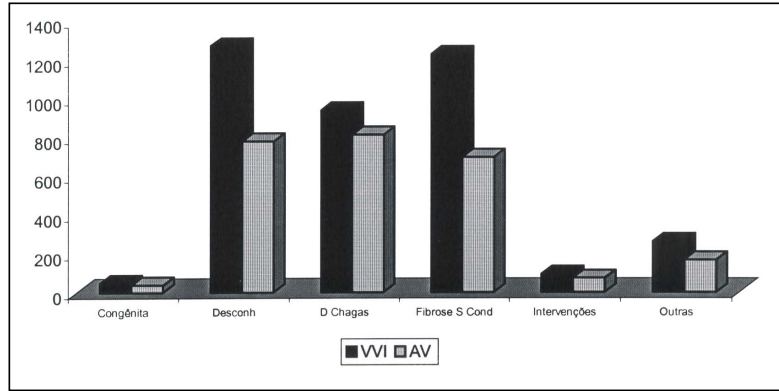

Fig. 10 - Modos de estimulação utilizados nos implantes iniciais, agrupados segundo a etiologia do distúrbio da condução dos pacientes.

sistema de condução, em $72(2,1 \%)$ foram citadas intervenções médicas, e em $166(4,8 \%)$, outras causas foram relatadas (Figura 10).

\section{COMENTÁRIOS}

No presente estudo foi possível verificar que, no Brasil, no ano de 1999, a tendência de diminuição da utilização de marcapassos ventriculares verificada nos anos anteriores persistiu. No presente estudo a relação entre implantes ventriculares e atrioventriculares (VVI/AV) foi de 1, 3:1 para os implantes iniciais. Quando foi optado por modos fisiológicos, a grande maioria dos pacientes recebeu marcapasso atrioventricular, sendo que apenas $0,7 \%$ dos implantes foram atriais puros.

Dentre os fatores que influenciaram a escolha do modo de estimulação, pudemos identificar nos dados enviados ao RBM: 1) a região onde o hospital está instalada; 2) a idade; 3) a classe funcional e 4) o distúrbio de condução do paciente.

\section{Diferenças Regionais}

Quando se analisa a região do Brasil onde o implante foi realizado, observa-se que à exceção da região centro-oeste, em todas as demais regiões implantaram-se mais marcapassos ventriculares. $\mathrm{Na}$ região sudeste a relação $\mathrm{VVI} / \mathrm{AV}$ foi de $1,3: 1$, na nordeste de 1,4:1, na sul de 2,0:1 e na região norte foi 1,9: 1. Na região centro-oeste, o número de implantes atrioventriculares foi maior que o número de ventriculares com uma relação de 1,5 duplacâmara para cada ventricular.

\section{Idade}

A idade do paciente influenciou fortemente a escolha do modo de estimulação.
Nas crianças e adolescentes houve uma tendência a serem utilizados marcapassos ventriculares em maior número que os atrioventriculares (relação VVI / $A V=1,3: 1)$

$\mathrm{Na}$ população adulta jovem, 21 a 40 anos, o número de implantes atrioventriculares foi maior que o de ventriculares, com relação $A V / V V I=1,7: 1)$. Da mesma forma, na faixa etária entre 41 e 60 anos, implantou-se mais marcapassos de duplacâmera (relação AV / VVI = 1,3:1).

No caso da população sexagenária e septuagenária implantou-se mais marcapassos ventriculares, com relação VVI / $\mathrm{AV}=1,4: 1$.

Para os octogenários e nonagenários o modo ventricular foi o mais escolhido (relação VVI / AV = 3,2:1.

Estes dados estão em concordância com as conclusões apresentadas por JAHANGIR et al. (8), em 1999, que, estudando retrospectivamente 432 pacientes octogenários e nonagenários, demonstraram que a escolha do modo de estimulação, ventricular ou atrioventricular, não interferiu na mortalidade desse grupo de pacientes. Segundo esses autores, a sobrevida foi modificada por doenças associadas, pela classe funcional para ICC, pela função ventricular e pela idade do paciente no momento do implante ${ }^{(8)}$.

\section{Classe Funcional}

A classe funcional influenciou a escolha do modo de estimulação fazendo com que pacientes sem insuficiência cardíaca recebessem marcapassos fisiológicos numa proporção maior que os pacientes com sintomas (classes II, III e IV).

$\mathrm{Na}$ classe funcional I a relação AV / VVI foi de $1,4: 1$; na classe II foi $0,8: 1$; na classe III foi de $0,7: 1$ e na classe IV, 0,5:1.

Esta tendência de utilizar marcapasso ventricular nos pacientes com maior grau de insuficiência cardíaca está em concordância com estudos hemodinâmicos realizados em pacientes chagásicos portadores de bloqueios avançados da condução atrioventricular, onde foi demonstrada piora ou ausência de melhora hemodinâmica, em pacientes com disfunção ventricular, quando da mudança da estimulação ventricular para atrioventricular, principalmente durante o exercício(11).

LINDE-EDELSTAM et al. ${ }^{(9)}$, em 1992, entretanto, em estudo prospectivo com 148 pacientes portadores de bloqueios atrioventriculares, randomizados para os modos VVI e VDD, demonstraram que a sobrevida dos pacientes foi semelhante à da população geral quando não há sinais de 
Costa R, Teno L A C, Groppo A A, Ávila Neto V, Beltrame R, Marques C P, Brofman P R S - Registro Brasileiro de Marcapassos: escolha do modo de estimulação no ano de 1999. Rev Bras Cir Cardiovasc 2000; 15(3): 263-71.

insuficiência cardíaca previamente ao implante, e que a mortalidade é influenciada pelo modo de estimulação apenas quando existe insuficiência cardíaca prévia.

\section{Distúrbio do Sistema Éxcito-Condutor}

O distúrbio do tecido de condução influenciou consideravelmente a escolha do modo de estimulação.

Pacientes portadores de doença do nó sinusal receberam aproximadamente duas vezes mais marcapassos fisiológicos que ventriculares (relação $\mathrm{AV} / \mathrm{VVI}=2,1: 1)$

Essa conduta está em concordância com a literatura. Na doença do nó sinusal, diversos estudos retrospectivos têm sugerido a maior sobrevida e a menor incidência de fibrilação atrial e insuficiência cardíaca congestiva nos portadores de marcapassos atriais e atrioventriculares. Esses estudos, entretanto, apresentam "vícios" nos critérios de seleção de modo, sendo o principal deles, que os pacientes portadores de marcapassos ventriculares são habitualmente os mais idosos e, muitas vezes, já portadores de fibrilação atrial ou doenças incuráveis no momento do implante do marcapasso $(7,10)$. ANDERSEN et al. (5), em 1997, em trabalho prospectivo e randomizado com 225 pacientes portadores de doença do nó sinusal, demonstraram que após cinco anos de seguimento, o implante de marcapasso atrial resultou em menor mortalidade total e cardiovascular, menor incidência de fibrilação atrial, de fenômenos tromboembólicos e de insuficiência cardíaca congestiva que os ventriculares.

Embora os pacientes portadores de bloqueio atrioventriculares tenham recebido implantes ventriculares em maior número, é possível verificar que a indicação de marcapassos bicamerais foi maior nos portadores de bloqueios do 2 o grau (relação VVI
/ $\mathrm{AV}=1,2: 1)$ do que nos portadores de bloqueios totais (relação VVI / $\mathrm{AV}=1,8: 1$ ).

É possível verificar que a grande maioria dos pacientes portadores de flutter ou fibrilação atrial receberam marcapassos ventriculares (relação VVI / AV $=10: 1$ ). Setenta e um pacientes, entretanto, receberam implantes atrioventriculares, provavelmente com o objetivo de prevenção da fibrilação atrial crônica.

\section{Outros Fatores Analisados}

Os sintomas pré-operatórios, a etiologia do distúrbio da condução e o sexo do paciente foram os outros aspectos estudados no presente relato. Não foram encontradas evidências de que esses parâmetros possam ter influenciado na escolha do modo de estimulação.

A análise dos dados obtidos pelo Registro Brasileiro de Marcapassos tem sido de grande utilidade para a observação da tendência dos profissionais brasileiros no tratamento dos pacientes portadores de bradiarritmias (12-26). O comportamento da escoIha do modo de estimulação pode ser observada com clareza nos dados obtidos em 1999.

Verifica-se que, a despeito das influências regionais, manifestadas pela especialização dos hospitais e mesmo dos médicos que realizam os procedimentos, normas rígidas, baseadas em evidências da literatura, têm sido aplicadas na escolha do modo de estimulação para os pacientes.

Modos de estimulação de dupla-câmara têm sido utilizados nos pacientes mais jovens, nos portadores de doença do nó sinusal e com menor grau de lesão do sistema His-Purkinje, onde o risco de síndrome do marcapasso é maior. Pacientes idosos, portadores de insuficiência cardíaca avançada e enfermos com bloqueio atrioventricular total receberam maior número de indicações para marcapassos ventriculares. 
Costa R, Teno L A C, Groppo A A, Ávila Neto V, Beltrame R, Marques C P, Brofman P R S - Registro Brasileiro de Marcapassos: escolha do modo de estimulação no ano de 1999. Rev Bras Cir Cardiovasc 2000; 15(3): 263-71.

RBCCV 44205-512

Costa R, Teno L A C, Groppo A A, Ávila Neto V, Beltrame R, Marques C P, Brofman P R S - Brazilian Pacemaker register: pacing mode choice in 1999. Rev Bras Cir Cardiovasc 2000; 15(3): 263-71.

ABSTRACT: Brazilian pacemaker register (RBM) is a nationwide database to collect data about all permanent pacemaker procedures performed in Brazil. It is a task force composed of the Health Ministry, Pacemaker Companies and Medical Society. This is a survey reporting the $6^{\text {th }}$ complete year of operation. From January $1^{\text {st }}, 1999$ to December $31^{\text {st }}, 1999,11.048$ surgical procedures for permanent cardiac pacing were performed: 8141 initial implants $(73.7 \%)$ and 2907 re-operations $(26.3 \%)$. Single chamber pacemakers were implanted in 6779 patients $(61.4 \%)$ and dual chamber in $4258(38.6 \%)$. Only 99 atrial single chamber pacemakers were implanted $(0.7 \%)$. It was possible to detect some factors that had influenced the pacing mode choice for ventricular (VVI) or dual chamber (DC) pacing: 1) the Brazilian region where the procedure was performed; 2) the age of the patient; 3 ) the functional class for Heart Failure (NYHA) and the preoperative EKG finding.

Regional Influence: Ventricular pacemakers were more prevalent in all regions except the central-west where the DC / VVI ratio was 1.5: 1. In all other regions the VVI / DC ratio ranged from 1.3 to 2.0: 1 .

Age: Atrioventricular pacemakers were preferred for patients from 21 to $60 \mathrm{yrs}$. (DC / VV ratio range = 1.7:1 and 1.3:1, respectively for ages 21 to 40 and 41 to $60 \mathrm{yrs}$.). In all other age classes ventricular pacing mode was prevalent

Heart Failure: Ventricular pacemakers were the choice in classes II, III and IV (VVI / DC ratio $=1.2: 1$ for class II, $1.5: 1$ for class III and 1.9: 1 in class IV). In class I patients, more dual chamber pacemakers were implanted $(\mathrm{DC} / \mathrm{VVI}$ ratio $=1.4: 1)$.

Pre-operative EKG Finding: Only sinus syndrome patients received more dual chamber pacemaker than ventricular (DC/VVI ratio $=2.1: 1)$. In all other types of conduction disturbances, more ventricular pacemakers were implanted than dual chamber: in $2^{\text {nd }}$ degree AV block the VVI / DC ratio was 1.2:1, in $3^{\text {rd }} \mathrm{AB}$ block it was 1.8: 1 and in atrial flutter / fibrillation patients 10: 1 .

Other Factors: Gender, preoperative symptoms and etiology of conduction disturbance were not related to pacing mode choice.

DESCRIPTORS: Pacemaker, artificial. Cardiac pacing, artificial, methods. Medical records, Brazil. Databases, Brazil. Pacemaker, artificial, data collection, Brazil. Cardiac pacing, artificial, implantation, data collection. Database management system, Brazil. Cardiac pacing, artificial, implantation, data collection. Brazilian Pacemaker Register .

\section{REFERÊNCIAS BIBLIOGRÁFICAS}

1 Andrade J C S, Ávila Neto V, Braile DM et al. - Diretrizes para o implante de marcapasso cardíaco permanente. Reblampa 1999; 12: 1-9.

2 Gregoratos G, Cheitlin M D, Conill A et al. - ACC/AHA Guidelines for Implantation of Cardiac Pacemakers and Antiarrhythmia Devices: executive summary - a report of the American College of Cardiology/American Heart Association Task Force on Practice Guidelines. Circulation 1998, 97: 1325-35.

3 Ray S G, Griffith M J, Jamieson S, Bexton R S, Gold R $G$ - Impact of the recommendations of the British Pacing and Electrophysiology Group on pacemaker prescription and on the immediate costs pacing in the Northern Region. Br Heart J 1992; 68: 531-4.

4 Sutton R \& Bourgeois I - Cost benefit analysis of single and dual chamber pacing for sick sinus syndrome and atrioventricular block: an economic sensitivity analysis of the literature. Eur Heart J 1996; 17: 574-82.

5 Andersen H R, Nielsen J C, Thomsen P E et al. - Long- term follow-up of patients from a randomised trial of atrial versus ventricular pacing for sick-sinus syndrome. Lancet 1997; 350: 1210-6.

6 Alpert M A, Curtis J J, Sanfelippo J F et al. - Comparative survival after permanent ventricular and dual chamber pacing for patients with chronic high degree atrioventricular block with and without preexistent congestive heart failure. J Am Coll Cardiol 1986; 7: 925-32.

7 Alpert M A, Curtis J J, Sanfelippo J F et al. - Comparative survival following permanent ventricular and dualchamber pacing for patients with chronic symptomatic sinus node dysfunction with and without congestive heart failure. Am Heart J 1987; 113: 958-65.

8 Jahangir A, Shen W K, Neubauer S A et al. - Relation between mode of pacing and long-term survival in the very elderly. J Am Coll Cardiol. 1999; 33: 1208-16.

9 Linde-Edelstam C, Gullberg B, Norlander R, Pehrsson S $\mathrm{K}$, Rosenqvist $\mathrm{M}$, Ryden $\mathrm{L}$ - Longevity in patients with high degree atrioventricular block paced in the atrial synchronous or the fixed rate ventricular inhibited 
Costa R, Teno L A C, Groppo A A, Ávila Neto V, Beltrame R, Marques C P, Brofman P R S - Registro Brasileiro de Marcapassos: escolha do modo de estimulação no ano de 1999. Rev Bras Cir Cardiovasc 2000; 15(3): 263-71.

mode. Pacing Clin Electrophysiol 1992; 15: 304-13.

10 Tung R T, Shen W K, Hayes D L, Hammill S C, Bailey K R, Gersh R J - Long-term survival after permanent pacemaker implantation for sick sinus syndrome. Am J Cardiol 1994; 74:1016-20.

11 Costa R - Contribuição ao estudo da estimulação ventricular e da atrioventricular universal em portadores da miocardiopatia chagásica: avaliação clínica e hemodinâmica em repouso e exercício. [Tese de Doutorado] São Paulo: Faculdade de Medicina da Universidade de São Paulo, 1990.

1 Costa R \& Leão, M I P - Registro Brasileiro de Marcapassos. Rev Bras Marcapasso Arritmia, 1993; 6: 31-4.

13 Costa R \& Leão M I P. Implantação do Registro Brasileiro de Marcapassos. Rev Bras Marcapasso Arritmia 1994; 7: 2-3.

14 Costa R, Leão M I P, Latini R - Implantação do Registro Brasileiro de Marcapassos. Rev Bras Marcapasso Arritmia 1994; 7: 72-7.

15 Costa R \& Leão M I P - Registro Brasileiro de Marcapassos: Resultados preliminares. Rev Bras Marcapasso Arritmia 1994; 7: 124-9.

16 Costa R \& Leão M I P - Registro Brasileiro de Marcapassos: resultados obtidos no quadrimestre setembro/dezembro de 1994. Reblampa, 1995; 8: 22-9.

17 Costa R \& Leão M I P - Registro Brasileiro de Marcapassos: resultados obtidos no primeiro quadrimestre de 1995. Reblampa 1995; 8: 99-106.

18 Costa R \& Leão M I P - Registro Brasileiro de Marcapassos: resultados obtidos no segundo quadrimestre de 1995: aspectos atuais da escolha dos modos de estimulação no Brasil. Reblampa 1995; 8: 272-9.
19 Costa R \& Leão M I P - Registro Brasileiro de Marcapassos: resultados obtidos no terceiro quadrimestre de 1995: aspectos regionais da escoIha dos modos de estimulação cardíaca permanente no Brasil. Reblampa 1996; 9: 37-42.

1 Leão M I P \& Costa R - Registro Brasileiro de Marcapassos: resultados do primeiro ano de implantação do sistema. Arq Bras Cardiol 1995; 65 (supl.1): 43.

21 Costa R \& Leão M I P - Experiência Brasileira com o uso de marcapasso cardíaco artificial: resultados atuais obtidos pelo Registro Brasileiro de Marcapassos RBM. Rev Bras Cir Cardiovascular 1995; 10: 84-9.

22 Leão M I P \& Costa R - Brazilian Pacemaker Registry. Eur JCPE 1996; 6 (1 Suppl. 5): 169 (Abstract).

23 Leão M I P, Costa R, Pachón M J C et al. - Registro Brasileiro de Marcapassos no ano de 1995: análise do perfil de pacientes chagásicos e não chagásicos. Reblampa 1996; 9: 75-82.

24 Costa, R - Influência do grau funcional para insuficiência cardíaca congestiva nas indicações de implante de marcapasso cardíaco permanente: apresentação dos dados do Registro Brasileiro de Marcapassos referentes ao ano de 1996. Reblampa 1998; 11: 127-34.

25 Costa, R - Análise das características clínicas dos pacientes submetidos a implante inicial e a reoperações: apresentação dos dados do Registro Brasileiro de Marcapassos (RBM) referente ao ano de 1997. Reblampa 1999; 12: 121-7.

26 Costa, R - Escolha do modo de estimulação no Brasil: análise das características dos pacientes submetidos a implantes ventriculares e atrioventriculares a partir dos dados do Registro Brasileiro de Marcapassos (RBM) no ano de 1998. Reblampa 2000; 13: 49-57. 